\title{
LOCAL RELIABILITY BASED SENSITIVITY ANALYSIS WITH THE MOVING PARTICLES METHOD
}

\author{
CARSTEN PROPPE ${ }^{1}$ \\ ${ }^{1}$ Karlsruhe Institute of Technology, Chair of Engineering Mechanics \\ Kaiserstr. 10, Bdg. 10.23, 76131 Karlsruhe, Germany \\ proppe@kit.edu
}

Key words: Local Sensitivity Analysis, Reliability, Multilevel Splitting, Moving Particles

\begin{abstract}
Local reliability sensitivity methods aim at determining the partial derivatives of the failure probability or the reliability index with respect to model parameters. For efficient local reliability based sensitivity analysis, it is important to avoid repeated evaluations of the performance function. To this end, an extension of the moving particles method to local reliability based sensitivity analysis is presented that is completely based on the already evaluated samples for the reliability estimate and thus avoids repeated evaluations of the performance function. In order to further reduce the variance of the estimator and to increase the efficiency, a multilevel variant of the estimator is proposed. The method is discussed in detail and illustrated by means of examples.
\end{abstract}

\section{INTRODUCTION}

Reliability based sensitivity analysis investigates the dependence of the failure probability on model parameters. In this study, the relevant model parameters are distribution parameters related to the probabilistic characterization of the model input. For sensitivity analysis, local and global approaches can be distinguished. Local reliability sensitivity analysis methods compute the partial derivatives of the failure probability or the reliability index with respect to model parameters. Global reliability sensitivity analysis aims at determining the influence of a model parameter on the failure probability over the entire range of possible values for this parameter. It is often based on Sobol' indices.

In [11], local reliability based sensitivity analysis is considered and partial derivatives of the failure probability by means of importance sampling are computed without any additional evaluations of the performance function. This approach is extended in [9] to subset simulation by computing partial derivatives of the conditional probabilities for each of the subsets.

Here, the reliability analysis is carried out by the moving particles method, cf. [10], where a threshold is associated to each sample, samples are moved to new positions in the design space and the number of moves for the initial samples (the particles) to reach the failure region are counted and yield an estimator for the failure probability, which is based on a Poisson process. The estimator is of comparable accuracy and efficiency as the subset simulation estimator. For local reliability based sensitivity analysis, it is important to avoid repeated evaluations of the performance function. To this end, an extension of the 
moving particles method to local reliability based sensitivity analysis is presented. It is shown that the method is not only completely based on the already evaluated samples for the reliability estimate and thus avoids repeated evaluations of the performance function, but that it can be carried out after the last move of a particle and just once after the reliability estimation has been carried out.

The paper is organized in five sections. After the introduction, a brief outline of the moving particles method is given. Following this, local reliability sensitivity analysis is integrated in the moving particles method and it is shown that only a single postprocessing step is required which is carried out when the last particle has been moved into the failure domain. Finally, the accuracy and efficiency of the proposed method is investigated by examples and conclusions are drawn.

\section{BRIEF OUTLINE OF THE MOVING PARTICLES METHOD}

Structural reliability analysis is concerned with the computation of the failure probability

$$
P_{F}=\int_{g(x)<0} p(x, \theta) \mathrm{d} x
$$

where the real valued function $g(x), x \in \mathbb{R}^{n}$, denotes the performance function of the structure, $p(x, \theta)$ is the probability density function of those structural parameters that are assumed to be random variables and $\theta$ contains the parameters of the probability distributions. The limit state function $g(x)=0$ separates


the safe domain applications, the failure probability a huge number of evaluations of

In order to reduce the computational effort, impo
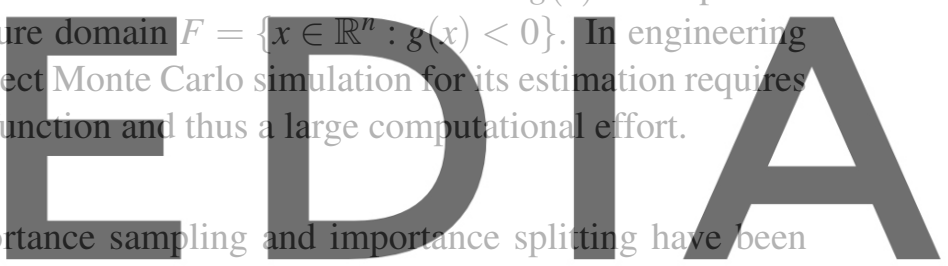

widely applied to the estimation of rare events [8]. Importance sampling estimates the occurrence of rare

Register foreptse generating samples from analternative distribution and correcting for the bias butheintroduci-ermark tion of weights. The success of this method relies on the quality of the importance sampling density, which is efficiently constructed in an adaptive way.

Importance splitting allows estimating small failure probabilities efficiently, even for problems that involve a high-dimensional vector of input random variables [8]. The general idea of importance splitting is to discard samples that are too far away from a threshold and to regenerate new samples from the remaining ones. In order to maintain the independence of the samples, Markov Chain Monte Carlo simulation starting from remaining samples is frequently employed.

For time-independent problems, subset simulation [1] is a very efficient method for a wide range of applications. This method considers nested subsets generated by levels of the performance function. These nested subsets yield a multiplicative decomposition of the failure probability in larger conditional probabilities. For the estimation of these conditional probabilities, conditional samples are generated by means of Markov Chain Monte Carlo simulation. 
The moving particles algorithm [3] can be considered as a special case of subset simulation with a maximum number of subsets. It starts with an initial Monte Carlo simulation (MCS) with $N$ samples $x_{j}^{(1)} \in \mathbb{R}^{n}, j=1, \ldots, N$, distributed according to the probability density function $p(x, \theta)$. The values $g\left(x_{j}^{(1)}\right), j=1, \ldots, N$, of the $N$ samples are ranked. These initial samples (the particles) are then moved to the failure region by the following procedure: For the sample with the maximum value of the performance function (thus furthest away from a limit state), a Markov chain Monte Carlo simulation (MCMC) is carried out starting from one of the other samples and the next state of the Markov chain is accepted, if the value of the performance function can be reduced. The Markov chain can be generated either by application of the classical Metropolis-Hastings algorithm or by direct sampling from a normal transition kernel and requires a burn-in period in order to maintain independence of the samples. This algorithm is repeated and stops if all the particles have been moved into the failure domain. The total number of moves $M_{T}$ is counted.

Interpreted as a special case of subset simulation with a maximum number of domains, each move of a particle gives rise to a subset $F_{i}=\left\{x \in \mathbb{R}^{n}: g(x)<g_{i}\right\}, i=1, \ldots, M_{T}$, where $g_{i}=\max _{1 \leq j \leq N} g\left(x_{j}^{(i)}\right)$, $i=1, \ldots, M_{T}$, denotes the maximum value of the performance function of the $N$ particles $x_{j}^{(i)}, j=1, \ldots, N$, when carrying out the $i$ th move. For the conditional probability

$$
P_{i}=P\left(G<g_{i} \mid G<g_{i-1}\right),
$$

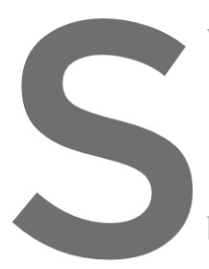

where for simplicity, the random variable $\mathrm{g}(X)$ is abbreviated $b$
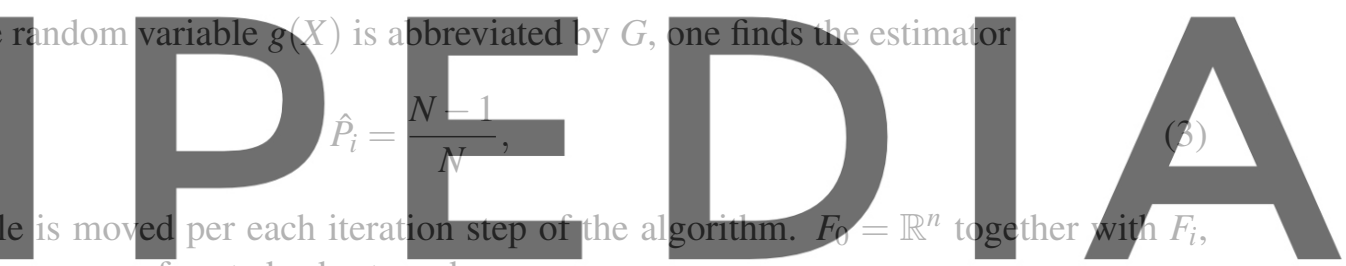

$i=1, \ldots, M_{T}$, is a finite sequence of nested subsets and

Register for free at https//www.scipedia.com to download the version without the watermark $F=\bigcap_{i=0} F_{i}$

Therefore, one finds for the failure probability

$$
P_{F}=\prod_{i=1}^{M_{T}} P\left(F_{i} \mid F_{i-1}\right)=\prod_{i=1}^{M_{T}} P_{i}
$$

and thus

$$
\hat{P}_{F}=\left(\frac{N-1}{N}\right)^{M_{T}}
$$

is an estimator for $P_{F}$.

In addition, this algorithm has the advantage that the number of moves of the particles is a Poisson distributed random variable that can be directly related to the failure probability $P_{F}$. Thus, properties of the Poisson distribution can be used to analyze the algorithm. For each initial sample $j, j=1, \ldots, N$, the 
number $M_{j}$ of moves until it reaches the failure region is counted. As has been shown in [3], the number of moves to get an initial sample into the failure region follows a Poisson distribution with parameter $\lambda=-\log P_{F}$. The estimator for the parameter of the Poisson distribution is obtained from $\lambda=E[M]$ as

$$
\hat{\lambda}=\frac{\sum_{j=1}^{N} M_{j}}{N}=\frac{M_{T}}{N} .
$$

In order to obtain an unbiased estimate, it is mandatory that the trajectories of the Poisson process generated from the initial samples remain independent until the samples finally reach the failure domain. In [10], two means are proposed to maintain the independence:

- Burn-in: The Markov chain simulation is carried out with a burn-in period. The burn-in should ensure the independence of the candidate and the seed of the Markov chain.

- Seed avoidance: Repeated use of the same starting point for the Markov chain should be avoided. Once a sample has been used as starting point, the sample and its offspring should not be used as starting point again.

\section{LOCAL RELIABILITY SENSITIVITY ANALYSIS WITH THE MOVING PARTICLES METHOD}

\subsection{Singlelevel version}

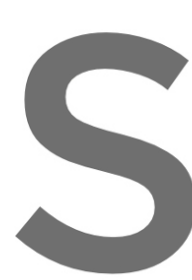

Applying the product 1

with respect to $\theta$ :

With
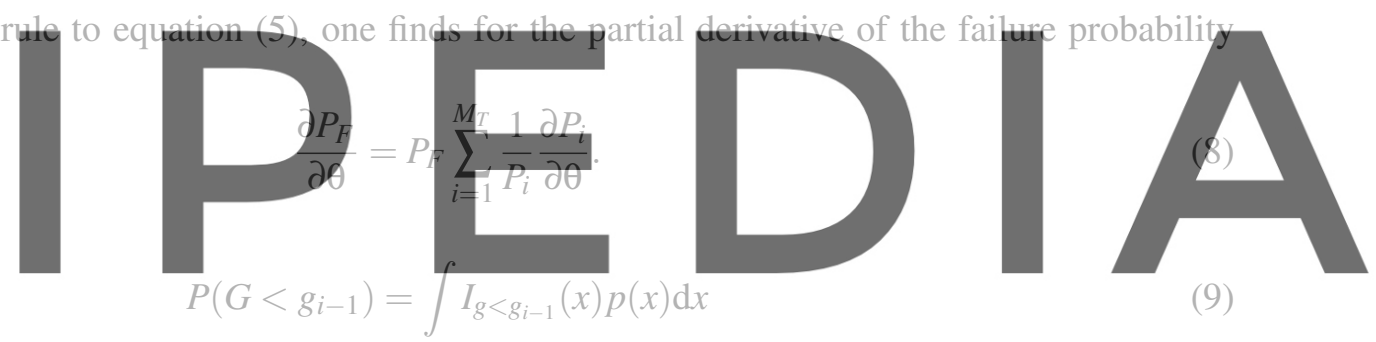

(9)

Register forffree at https//www.scipedia.com to download the version without the watermark

$$
P_{i}=P\left(G<g_{i} \mid G<g_{i-1}\right)=\int I_{g<g_{i}}(x) \frac{I_{g<g_{i-1}}(x) p(x)}{P\left(G<g_{i-1}\right)} \mathrm{d} x,
$$

where the indicator function is given by

$$
I_{g<g_{i}}(x)= \begin{cases}1, & \text { if } g(x)<g_{i}, \\ 0, & \text { otherwise }\end{cases}
$$

the partial derivative of the conditional probability $P_{i}$ is given by

$$
\begin{aligned}
\frac{\partial P_{i}}{\partial \theta} & =\frac{\partial}{\partial \theta} \int I_{g<g_{i}}(x) \frac{I_{g<g_{i-1}}(x) p(x)}{P\left(G<g_{i-1}\right)} \mathrm{d} x \\
& =\int I_{g<g_{i}}(x) \frac{I_{g<g_{i-1}}(x)}{P\left(G<g_{i-1}\right)} \frac{\partial p(x)}{\partial \theta} \mathrm{d} x-\int I_{g<g_{i}}(x) \frac{I_{g<g_{i-1}}(x) p(x)}{P\left(G<g_{i-1}\right)^{2}} \frac{\partial P\left(G<g_{i-1}\right)}{\partial \theta} \mathrm{d} x \\
& =\int I_{g<g_{i}}(x) \frac{1}{P\left(G<g_{i-1}\right)} \frac{\partial p(x)}{\partial \theta} \mathrm{d} x-\int I_{g<g_{i}}(x) \frac{p(x)}{P\left(G<g_{i-1}\right)^{2}} \frac{\partial P\left(G<g_{i-1}\right)}{\partial \theta} \mathrm{d} x,
\end{aligned}
$$


as $g_{i}<g_{i-1}$ and thus $I_{g<g_{i}}(x) I_{g<g_{i-1}}(x)=I_{g<g_{i}}(x)$. The last expression can also be written as

$$
\frac{\partial P_{i}}{\partial \theta}=E_{G<g_{i-1}}\left[I_{g<g_{i}}(x) \frac{\partial \ln p(x)}{\partial \theta}\right]-\frac{P_{i}}{P\left(G<g_{i-1}\right)} \frac{\partial P\left(G<g_{i-1}\right)}{\partial \theta} .
$$

The expression in the last term involves the partial derivative of the probability related to a set of larger bound, namely $F_{i-1}$. Analogously to equation (8), we find the expression

$$
\frac{\partial P\left(G<g_{i-1}\right)}{\partial \theta}=P\left(G<g_{i-1}\right) \sum_{j=1}^{i-1} \frac{1}{P_{j}} \frac{\partial P_{j}}{\partial \theta}
$$

for this partial derivative. In summary, the expression for the partial derivative of the conditional probability $P_{i}$ with respect to $\theta$ reads

$$
\frac{\partial P_{i}}{\partial \theta}=E_{G<g_{i-1}}\left[I_{g<g_{i}}(x) \frac{\partial \ln p(x)}{\partial \theta}\right]-\sum_{j=1}^{i-1} \frac{1}{P_{j}} \frac{\partial P_{j}}{\partial \theta} .
$$

Inserting this expression into equation (8) and observing that $\hat{P}_{1}=\hat{P}_{2}=\ldots=\hat{P}_{M_{T}}$ yields a telescoping sum, such that the estimator for the sensitivity of the failure probability is obtained as
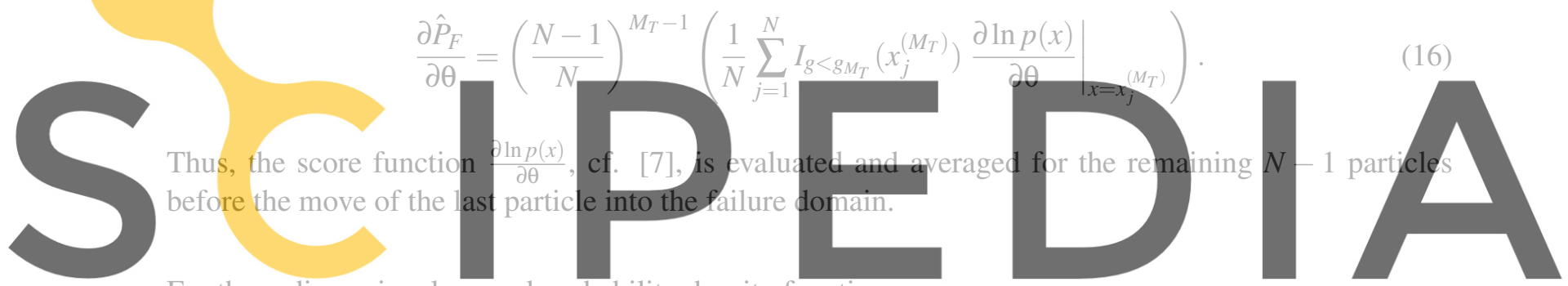

For the $n$-dimensional normal probability density function

\section{Register for free at https//wwww.scipedia.c®m to dowriload the version without the watermark}

the partial derivatives with respect to $\mu$ and $\Sigma$ yield the expressions

$$
\frac{\partial \ln p(x, \mu, \Sigma)}{\partial \theta}=\Sigma^{-1}(x-\mu)
$$

for $\theta=\mu$ and

$$
\frac{\partial \ln p(x, \mu, \Sigma)}{\partial \theta}=-\Sigma^{-1}+\frac{1}{2}\left(\Sigma^{-1} \circ I\right)+\Sigma^{-1}(x-\mu)(x-\mu)^{T} \Sigma^{-1}-\frac{1}{2}\left(\Sigma^{-1}(x-\mu)(x-\mu)^{T} \Sigma^{-1} \circ I\right)
$$

for $\theta=\Sigma$, where $\circ$ denotes the Hadamard product and $I$ the $n \times n$ identity matrix. 
Table 1: Distribution parameters for the random variables in example 1.

\begin{tabular}{ccccccc}
\hline & $q[\mathrm{~N} / \mathrm{m}]$ & $\ell[\mathrm{m}]$ & $A_{C}\left[\mathrm{~m}^{2}\right]$ & $E_{C}\left[\mathrm{~N} / \mathrm{m}^{2}\right]$ & $A_{S}\left[\mathrm{~m}^{2}\right]$ & $E_{S}\left[\mathrm{~N} / \mathrm{m}^{2}\right]$ \\
\hline mean value & 20000 & 12 & 0.04 & $2 \times 10^{10}$ & $9.82 \times 10^{-4}$ & $1 \times 10^{11}$ \\
std. dev. & 1400 & 0.12 & 0.0048 & $1.2 \times 10^{9}$ & $5.892 \times 10^{-5}$ & $6 \times 10^{9}$ \\
\hline
\end{tabular}

\subsection{Multilevel version}

Due to the rather low number of particles on which the estimator is built, the coefficient of variation can be rather high. In this regard, a multilevel approach [5] helps to reduce the coefficient of variation and thus to further improve the efficiency of the sensitivity analysis. To this end, consider a series of approximations $\left\{g_{i}(x)\right\}, i=0, \ldots, n$, for the performance function $g(x)$, where the index $i$ is related to an approximation parameter $h$ (e.g. stepsize, mesh parameter) and $g_{n}(x)=g(x)$.

The expectation

$$
E_{G<g_{M_{T}-1}}\left[I_{g<g_{M_{T}}} \frac{\partial \ln p(x)}{\partial \theta}\right]
$$

that has to be estimated in (16) is written as a telescoping sum

$$
E_{G_{0}<g_{M_{T}-1}}\left[I_{g_{0}<g_{M_{T}}} \frac{\partial \ln p(x)}{\partial \theta}\right]+\sum_{i=1}^{n} E_{G_{i-1}<g_{M_{T}-1}}\left[I_{g_{i}<g_{M_{T}}} \frac{\partial \ln p(x)}{\partial \theta}-I_{g_{i-1}<g_{M}} \frac{\partial \ln p(x)}{\partial \theta}\right] .
$$

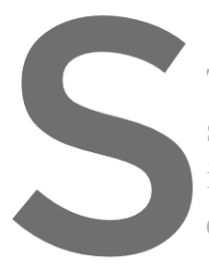

The idea of the multilevel method
scoping sum separately. This meth
is lower than that of the singleleve
expensive performance function $g_{n}$
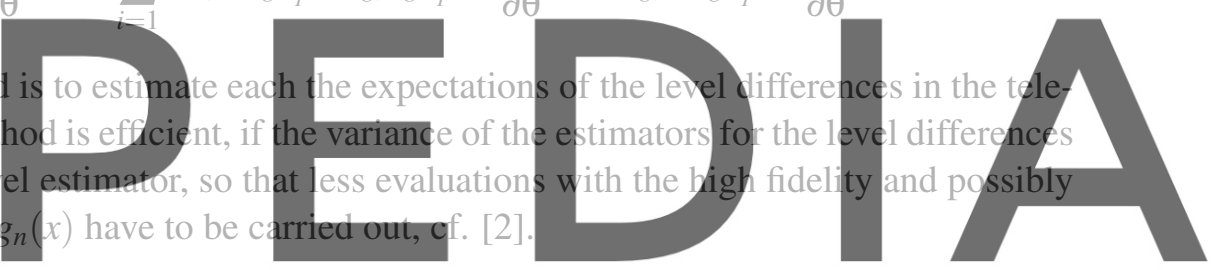

Register för free at https//www.scipedia.com to download the version without the watermark

\subsection{Example 1: Roof truss}

Consider the truss structure shown in Figure 1, cf. also [9]. The top boom and the compression bars are made of reinforced concrete (cross section area $A_{C}$, Young's modulus $E_{C}$ ), while the bottom boom and the tension bars are made of steel (cross section area $A_{S}$, Young's modulus $E_{S}$ ). The concentrated load $P$ represents a constant distributed load $q$ that is applied on the roof truss and thus $P=\frac{q \ell}{4}$. The parameters $q, \ell, A_{C}, E_{C}, A_{S}$ and $E_{S}$ are assumed to be independent normally distributed random variables. Table 1 displays the mean values and standard deviation of the random variables which are the same as in [9].

If the displacement of the top node should not exceed $3 \mathrm{~cm}$, the limit state function reads

$$
g\left(q, \ell, A_{C}, E_{C}, A_{S}, E_{S}\right)=0.03-\frac{q \ell^{2}}{2}\left(\frac{3.81}{A_{C} E_{C}}+\frac{1.13}{A_{S} E_{S}}\right)
$$

Table 2 summarizes the results for the estimated failure probability and the obtained sensitivities with respect to the distribution parameters. In order to be able to compare the influence of the parameters, it 


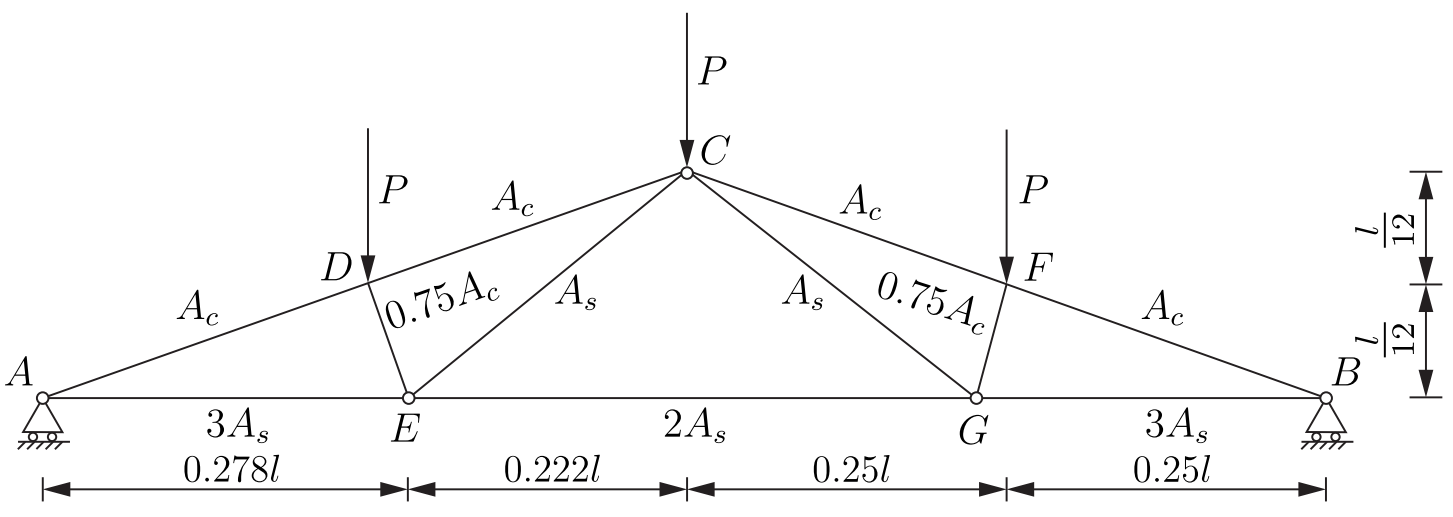

Figure 1: Roof truss structure.

also states the elasticity, which is given by

$$
\frac{\theta}{P_{F}} \frac{\partial P_{F}}{\partial \theta}
$$

cf. [4]. The initial number of samples, i.e. the particles, has been set to 2000. The relative error has been computed with respect to results obtained by Monte Carlo simulation and published in [9].

The results indicate that the mean values, notably the mean length $\ell$ and the mean distributed force $q$ are most influential on the failure probability. The approximation error is rather low. The proposed method based on just 2000 sanples is as exact as a Monte demonstrates the efficiency of the proposed method. are rather low. However, it was noted that for the the length $\ell$ and the Young's modulus of concrete $E$
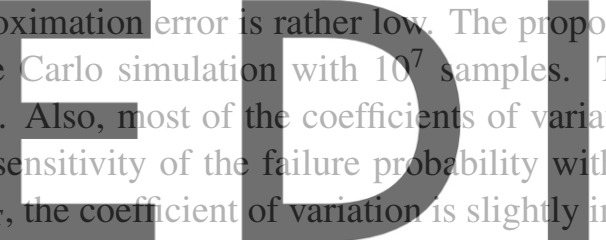

possible reason could be the fact that the proposed method computes an average on the 2000 samples,

Register for free at https//www.scipedia.com to download the version without the watermark

\subsection{Example 2: One storey one bay elastoplastic frame}

This example deals with a series system consisting of four failure modes that lead to nearly the same first oder reliability index $\beta$. Consider the elastoplastic frame structure shown in Figure 2. The four potential failure modes yield the performance functions

$$
\begin{aligned}
& g_{1}=2 M_{1}+2 M_{3}-4.5 S, \\
& g_{2}=2 M_{1}+M_{2}+M_{3}-4.5 S, \\
& g_{3}=M_{1}+M_{2}+2 M_{3}-4.5 S, \\
& g_{4}=M_{1}+2 M_{2}+M_{3}-4.5 S,
\end{aligned}
$$

cf. [9]. The performance function is thus given by the minimum of these four functions:

$$
g\left(M_{1}, M_{2}, M_{3}, S\right)=\min \left(g_{1}, g_{2}, g_{3}, g_{4}\right) .
$$


Table 2: Example 1: Failure probability and sensitivity of the failure probability with respect to the distribution parameters.

\begin{tabular}{cccccccc}
\hline & $P_{F}$ & $\frac{\partial P_{F}}{\partial m_{q}}$ & $\frac{\partial P_{F}}{\partial m_{\ell}}$ & $\frac{\partial P_{F}}{\partial m_{A_{C}}}$ & $\frac{\partial P_{F}}{\partial m_{E_{C}}}$ & $\frac{\partial P_{F}}{\partial m_{A_{S}}}$ & $\frac{\partial P_{F}}{\partial m_{E_{S}}}$ \\
\hline estimate & 0.00937 & $1.10 \mathrm{e}-5$ & 0.0403 & -2.110 & $-3.71 \mathrm{e}-12$ & -186 & $-1.81 \mathrm{e}-12$ \\
elasticity & & 23.5 & 51.9 & -9.1 & -8.0 & -19.6 & -19.5 \\
c.o.v. & 0.05 & 0.05 & 0.08 & -0.06 & -0.08 & -0.07 & -0.06 \\
rel. error [\%] & 0.5 & 0.7 & 0.9 & 1.0 & 1.2 & 0.1 & 0.6 \\
\hline \multirow{2}{*}{ estimate } & & $\frac{\partial P_{F}}{\partial \sigma_{q}}$ & $\frac{\partial P_{F}}{\partial \sigma_{\ell}}$ & $\frac{\partial P_{F}}{\partial \sigma_{A_{C}}}$ & $\frac{\partial P_{F}}{\partial \sigma_{E_{C}}}$ & $\frac{\partial P_{F}}{\partial \sigma_{A_{S}}}$ & $\frac{\partial P_{F}}{\partial \sigma_{E_{S}}}$ \\
\hline elasticity & $1.57 \mathrm{e}-5$ & 0.0182 & 2.5047 & $1.93 \mathrm{e}-12$ & 204 & $1.99 \mathrm{e}-12$ \\
c.o.v. & 2.4 & 0.2 & 1.3 & 0.2 & 1.3 & 1.3 \\
rel. error [\%] & 0.06 & 0.22 & 0.08 & 0.20 & 0.09 & 0.09 \\
\hline
\end{tabular}

$M_{i}, i=1, . ., 3$ and $S$ are assumed to be independent normally distrbuted random variables. For $M_{i}$, $i=1, \ldots, 3$, a mean value of 5.2872 and a standard deviation of 0.1492 is assumed, while for $S$, the mean value was 3.8378 and the standard deviation 0.385 modes is 3.334 for the $\mathrm{f}$
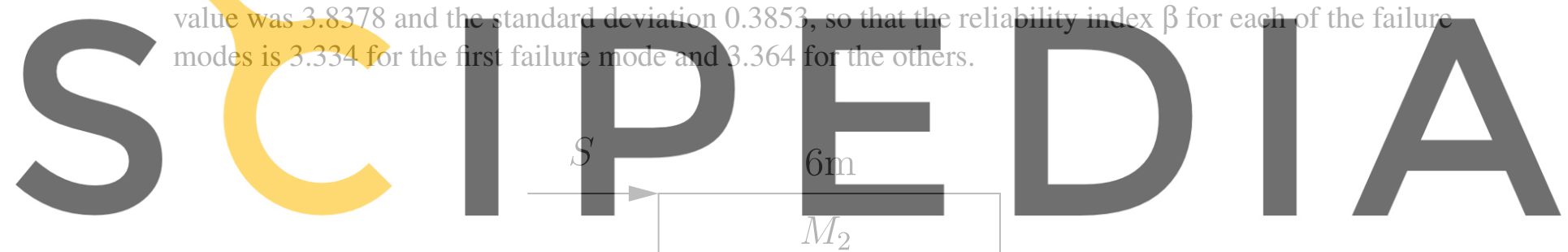

Register for free at https//www.scipedia.com to download the version without the watermark
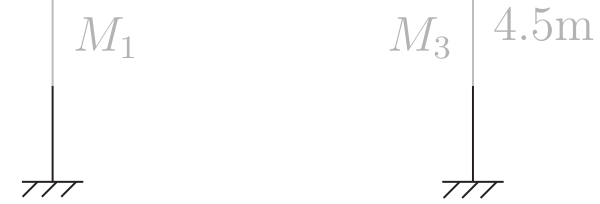

Figure 2: One storey one bay elastoplastic frame.

The results for the estimated failure probability and the obtained sensitivities are collected in Table 3 . The initial number of samples, i.e. the particles, has been set to 2000. Again, the relative error has been computed with respect to Monte Carlo simulation results with $10^{7}$ samples published in [9].

As Table 3 demonstrates, the mean values and the standard deviation related to the applied force $S$ influence the failure probability most. The approximation error is always less than $2 \%$. This confirms the findings of the previous example that the proposed method is very efficient and as exact as a highly accurate Monte Carlo simulation with $10^{7}$ samples. As in the previous example, some of the coefficients 
Table 3: Example 2: Failure probability and sensitivity of the failure probability with respect to the distribution parameters.

\begin{tabular}{lccccc}
\hline & $P_{F}$ & $\frac{\partial P_{F}}{\partial m_{M_{1}}}$ & $\frac{\partial P_{F}}{\partial m_{M_{2}}}$ & $\frac{\partial P_{F}}{\partial m_{M_{3}}}$ & $\frac{\partial P_{F}}{\partial m_{S}}$ \\
\hline estimate & 0.0182 & -0.0387 & -0.0236 & -0.038 & 0.1132 \\
elasticity & & -11.2 & -6.9 & -11.0 & 23.9 \\
c.o.v. & 0.05 & -0.11 & -0.18 & -0.13 & 0.04 \\
rel. error [\%] & 0.4 & 1.4 & 1.3 & 0.5 & 0.1 \\
\hline & & $\frac{\partial P_{F}}{\partial \sigma_{M_{1}}}$ & $\frac{\partial P_{F}}{\partial \sigma_{M_{2}}}$ & $\frac{\partial P_{F}}{\partial \sigma_{M_{3}}}$ & $\frac{\partial P_{F}}{\partial \sigma_{S}}$ \\
\hline estimate & & 0.0177 & 0.0195 & 0.0171 & 0.2318 \\
elasticity & & 0.1 & 0.2 & 0.1 & 4.9 \\
c.o.v. & & 0.29 & 0.3 & 0.34 & 0.05 \\
rel. error [\%] & & 1.2 & 0.6 & 3.0 & 0.1 \\
\hline
\end{tabular}

Table 4: Example 3: Failure probability and sensitivity of the failure probability with respect to the distribution parameters. a) Uncorrelated random variables, b) Correlated random variables with $\rho=0.5$.
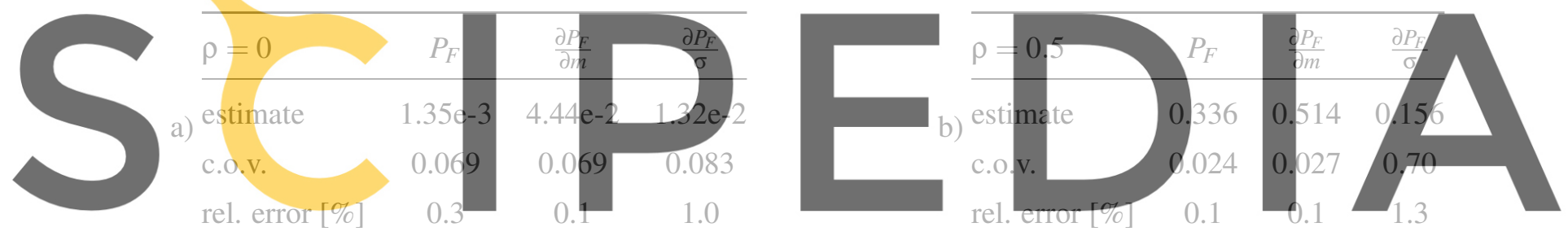

Register for free at https. www scipedia.com to download the version without the watermark

the parameters $\left.M_{i}, i=1, \ldots, 3\right)$.

\subsection{Example 3: High-dimensional problem}

This example deals with a component reliability problem of dimension 100. The performance function is given by

$$
g(X)=\frac{1}{1000+\sum_{i=1}^{100} X_{i}}-\frac{1}{1000+3 \sqrt{100}},
$$

where the 100 random variables are normal with mean $m=0$ and standard deviation $\sigma=1$, cf. [6]. Two cases are considered: (a) the random variables are independent, (b) all random variables are correlated with correlation coefficient $\rho=0.5$.

As Table 4 indicates, the high dimension of the vector of random parameters does not affect the accuracy 
of the obtained results. Also the correlation of the random variables seems to have no influence on the accuracy. However, it is observed that for the estimator of the partial derivative of the failure probability with respect to the standard deviation, the coefficient of variation is larger than for the uncorrelated case.

\subsection{Example 4: Multilevel method}

As a first test for the multilevel local reliability based sensitivity analysis, consider the performance functions

$$
g_{i}(X)=(3+(11-i) / 100)-X, i=1, \ldots, 10,
$$

for a standard normal random variable $X$. Figure 3 displays the relative errors and the standard deviation for the estimates of $P_{F}, \frac{\partial P_{F}}{\partial m}$ and $\frac{\partial P_{F}}{\partial \sigma}$. It is clearly seen that the three quantities converge to the single level result for $n=10$. The relative errors are of similar magnitude and decrease with nearly the same velocity. Moreover, the multilevel approach clearly reduces the standard deviation of the estimates. The standard deviation of the singlelevel estimator (which is approximately equal to that of the estimator of level 1) is much larger than that of the level differences.
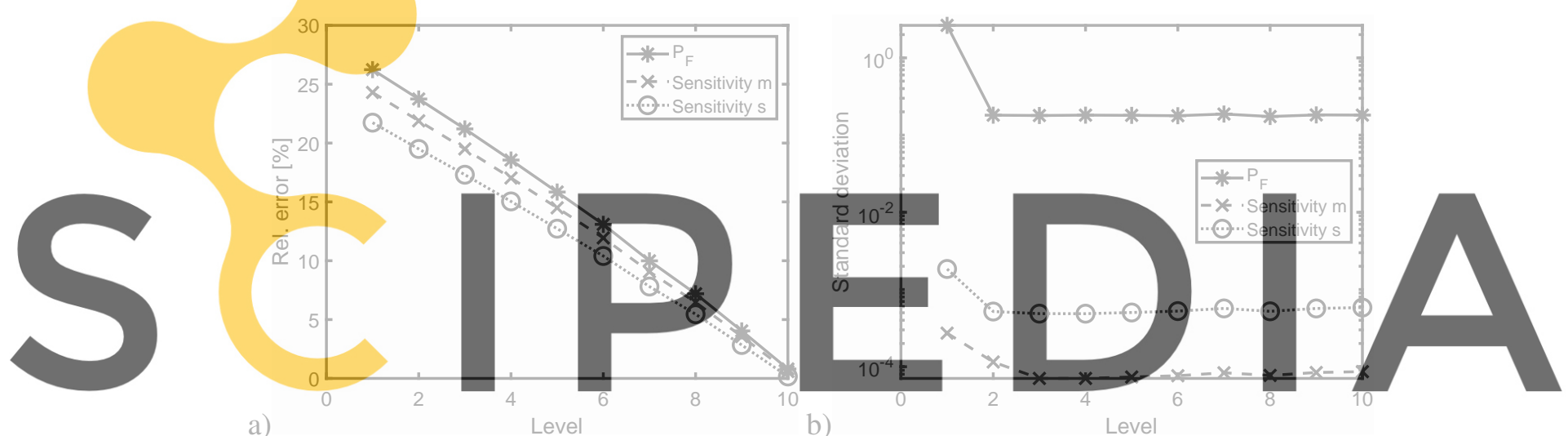

a)

b)

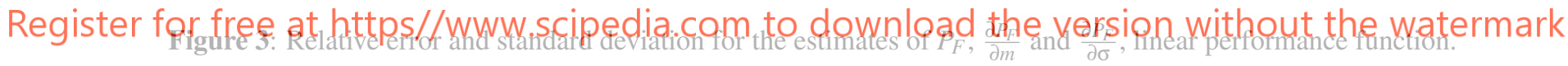

Next, the heat equation with random heat source is studied. Consider the linear stochastic partial differential equation

$$
\mathrm{d} u=\frac{\partial^{2} u}{\partial x^{2}} \mathrm{~d} t+\theta u \mathrm{~d} t+\sigma \mathrm{d} W_{t}
$$

with parameters $\theta$ and $\sigma$, where $\mathrm{d} W$ are the increments of a standard cylindrical Wiener process

$$
W_{t}=\sum_{k=1}^{\infty} W_{t}^{k} \sin (k \pi x)
$$

and $W_{t}^{k}$ are standard independent Brownian motions.

The boundary conditions are $u(x=0, t)=u(x=1, t)=0$ and the initial condition reads

$$
u(x, 0)=\sqrt{2} \sum_{k=1}^{\infty} \sin (k \pi x),
$$


so that the solution of the stochastic partial differential equation decouples and can be written as

$$
u(x, t)=\sqrt{2} \sum_{k=1}^{\infty} u_{k}(t) \sin (k \pi x)
$$

where the coefficients $u_{k}(t)$ are described by the linear stochastic ordinary differential equations

$$
\mathrm{d} u_{k}=\left(-\pi^{2} k^{2}+\theta\right) u_{k} \mathrm{~d} t+\sigma \mathrm{d} W_{t}^{k} .
$$

Their solutions at time $t$ are

$$
u_{k}(t)=\exp \left(\left(-\pi^{2} k^{2}+\theta\right) t\right)+\xi_{t}^{k},
$$

where $\xi_{t}^{k}$ is a normal random variable with mean zero and variance

$$
\frac{\sigma^{2}\left(1-\exp \left(2\left(-\pi^{2} k^{2}+\theta\right) t\right)\right)}{2\left(\pi^{2} k^{2}-\theta\right)}
$$

Failure is assumed to occur if $u(x=0.5, t=T)$ is larger than a given threshold. In the following, $\theta=1$, $\sigma=1$ and $T=1$ were set. The solution of the stochastic partial differential equation (28) is approximated by truncating the solution (31) at $k=k_{\max }$. Different values $i$ for $k_{\max }$ lead then to approximations $g_{i}(\xi)$ of the performance function $g(\xi)$.

Figure 4 displays the relative errors and the standard deviation for the estimates of $P_{F}, \frac{\partial P_{F}}{\partial m}$ and $\frac{\partial P_{F}}{\partial \sigma}$. Again, the relative error decreases quickly; however, it was noticed that the relative error for the sensitivity with respect to the mean value of the random variables is quite large for the first levels. Concerning the standard deviation, the example confirms the previous findings that the multilevel method is able to reduce the standard deviation of the estimates. In this example, the reduction is much larger for the estimates of the probability of failure than for the those of the sensitvities. For all three quantities, the standard deviation decreases with increasing number of levels.
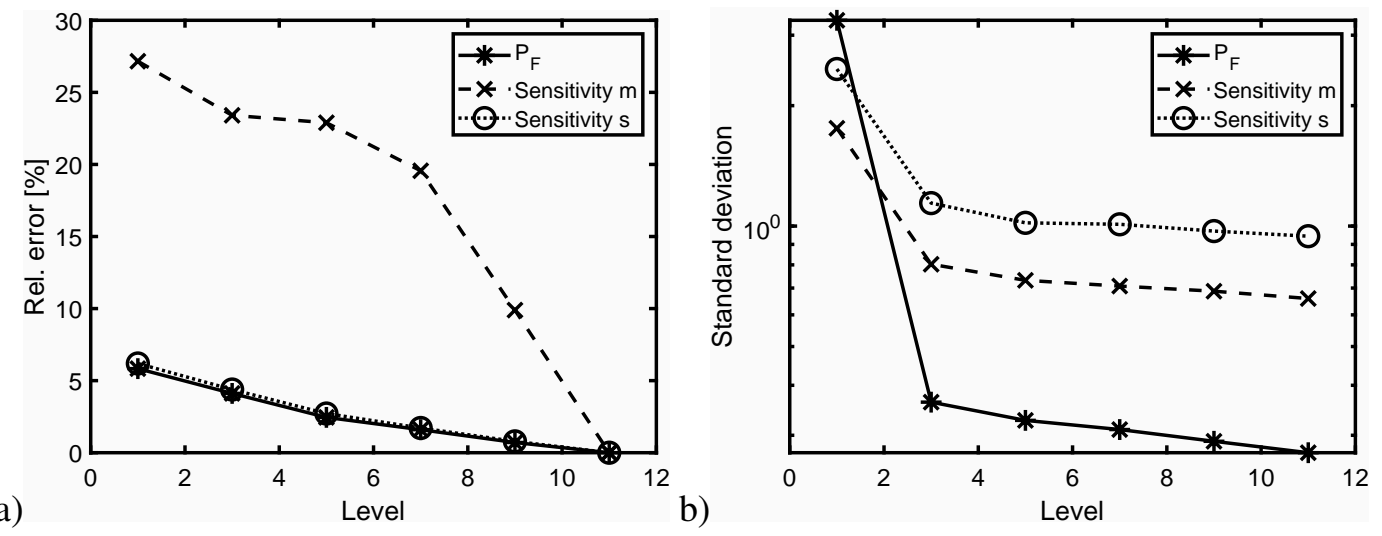

Figure 4: Relative error and standard deviation for the estimates of $P_{F}, \frac{\partial P_{F}}{\partial m}$ and $\frac{\partial P_{F}}{\partial \sigma}$, heat equation with random heat source. 


\section{CONCLUSIONS}

In this paper, local reliability based sensitivity analysis based on the moving particles method has been introduced. It has been shown that the sensitivity analysis can be carried out in a single postprocessing step after the last particle has been moved into the failure domain.

The examples demonstrate that the proposed method yields accurate estimates for the partial derivatives of the failure probability with respect to the distribution parameters of the random variables. However, due to the low number of particles on which the estimator is built, the coefficient of variation can be rather high. In this regard, the proposed multilevel method helps to reduce the standard deviation of the estimator and thus to further improve the efficiency of the sensitivity analysis.

\section{REFERENCES}

[1] S. K. Au and J. Beck. Important sampling in high dimensions. Structural Safety, 25:139-163, 2003.

[2] M. B. Giles. Multilevel Monte Carlo path simulation. Operations Research, 56:337-361, 2008.

[3] A. Guyader, N. Hengartner, and E. Matzner-Løber. Simulation and estimation of extreme quantiles and extreme probabilities. Applied Mathematics \& Optimization, 64:171-196, 2011.

[4] M. Lemaire. Structural Reliability. Wiley, 2010.

[5] C. Proppe. A multilevel moving particles method for reliability estimation. Probabilistic Engineering Mechanics, 59:to appear, 2020.

[6] S. Rahman. Stochastic sensitivity analysis by dimensional decomposition and score functions. Probabilistic Engineering Mechanics, 24:278-287, 2009.

[7] R. Rubinstein and A. Shapiro. Discrete Event Systems - Sensitivity Analysis and Stochastic Optimization by the Score Function Method. Wiley, 1993.

[8] G. Schuëller, H. Pradlwarter, and P. Koutsourelakis. A critical appraisal of reliability estimation procedures for high dimensions. Probabilistic Engineering Mechanics, 19(4):463-474, 2004.

[9] S. Song, Z. Lu, and H. Qiao. Subset simulation for structural reliability sensitivity analysis. Reliability Engineering and System Safety, 94:658-665, 2009.

[10] C. Walter. Moving particles: A parallel optimal multilevel splitting method with application in quantiles estimation and meta-model based algorithms. Structural Safety, 55:10-25, 2015.

[11] Y. T. Wu. Computational methods for efficient structural reliability and reliability sensitivity analysis. AIAA Journal, 32(8):1717-23, 1994. 